\title{
Climber Motion Optimization for the Tethered Space Elevator
}

\author{
Paul Williams ${ }^{*}$ and Wubbo Ockels ${ }^{\dagger}$ \\ Delft University of Technology, The Netherlands
}

\begin{abstract}
The tethered space elevator could provide a revolutionary means for enabling cheap transportation to geostationary altitude and beyond. Assuming that such a system can be built, one of the dynamic design problems is determining a means of moving the elevator along the tether so as to minimize the residual librational motion of the elevator ribbon and counterweight. In particular, this paper studies this problem from the point of view of dynamic optimization. A simplified dynamic model of the elevator system is derived that accounts for the fundamental libration mode and the motion of the elevator. The model is used to solve an optimal control problem that results in zero final librational motion of the ribbon.
\end{abstract}

\section{Introduction}

T HE tethered space elevator is a radical concept that has existed in the literature even prior to the modern spaceflight era [1]. The basic concept requires a tether or ribbon extending from geostationary altitude to the Earth's surface. However, in order to make the system feasible, the ribbon is required to extend past geostationary altitude. The system center of orbit is designed to remain at geostationary altitude so that the system spins in orbit at the same rate as the Earth rotates. This means that the tip of the ribbon is always located at the same location on the Earth's surface. Although Tsiolkovsky [1] is often credited with its invention, it was not until Pearson [2] that the concept was cemented in engineering terms. At the time of publication, no engineering material existed that could come close to the requirements needed for an Earth orbiting elevator. Recent developments in carbon nanotubes [3] offer one potential means for enabling the development of a full scale elevator system, albeit with many technical challenges to overcome.

Several previous works have dealt with various aspects of the dynamics of the elevator system. Cohen and Misra [4] derived a continuum model representation of the elevator ribbon to calculate the frequencies and mode shapes. Less rigorous estimation of frequencies and mode shapes has been presented elsewhere [5]. Williams [6] used a lumped mass model of the ribbon to calculate frequencies and mode shapes, and also simulated the effects of the passage of the elevator up to geostationary altitude. However, the elevator motion was prescribed according to an acceleration-constant speed-deceleration schedule. Cohen and Misra [7] used a simplified model of the elevator system to produce an analytic approximation for the libration response of the ribbon due to an elevator's motion under the assumption of constant velocity motion. They also showed that the use of multiple ascending elevators can be used to produce a zero net libration angle for the elevator ribbon. Studies of the dynamics of other elevator systems have also been undertaken, using models of varying fidelity [8-11].

If the elevator system is produced, it will be necessary to consider active control of the elevator during ascent, so that severe lateral oscillations are not induced. Furthermore, it is likely that motion planning will be required so as to reduce the resulting libration amplitude of the ribbon following passage of the elevator to the desired altitude. Present technology allows very rapid computation of optimal trajectories, which would enable planning of trajectories just prior to beginning ascent, or even real-time feedback control. This paper presents a numerical analysis of the optimal trajectories for the ascent of a climber to geostationary altitude. A simplified model of the system is used to produce the optimal trajectories, and a more detailed model is used to compare the results.

\footnotetext{
* Applied Researcher, Faculty of Aerospace Engineering, P.O. Box Postbus 5058, 2600 GB Delft, AIAA Member. E-mail: tethered.systems@gmail.com.

${ }^{\dagger}$ ASSET Chairholder, Faculty of Aerospace Engineering, P.O. Box Postbus 5058, 2600 GB Delft, E-mail: w.j.ockels@tudelft.nl.
} 


\section{Dynamic Elevator Model}

\section{A. Simplified Elevator Model}

To study the dynamics of the climber on the elevator system, detailed models of the system can be derived. However, to study the optimal means for controlling the climber to minimize the librations of the ribbon and counterweight, a simplified model of the system is used. The model treats only the in-plane motion of the ribbon. It considers the ribbon to be completely rigid, and the climber and counterweight to be point masses. The model derivation is a simplified version of the one presented in [7]. The reference system for the model is shown in Fig. 1. The origin of the rotating coordinate system is located at the Earth's surface at the anchor point. The counterweight, $m_{c}$, is located at a distance of $l$ from the anchor point, equal to the length of the ribbon. The elevator, $m_{e}$, is at a distance $d$ along the ribbon. The deviation of the ribbon from the vertical is measured by the libration angle $\theta$. The radius of the Earth is denoted by $R_{E}$.

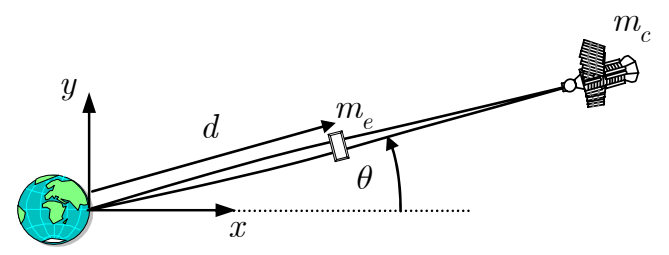

Fig. 1. Simplified representation of the tethered elevator system anchored to the Earth.

Let the position of the elevator, counterweight, and an arbitrary point along the ribbon at a distance $s$ from the anchor be written in the rotating coordinate frame as

$$
\begin{gathered}
\boldsymbol{r}_{e}=\left(R_{E}+d \cos \theta\right) \boldsymbol{i}+(d \sin \theta) \boldsymbol{j} \\
\boldsymbol{r}_{c}=\left(R_{E}+l \cos \theta\right) \boldsymbol{i}+(l \sin \theta) \boldsymbol{j} \\
\boldsymbol{r}(s)=\left(R_{E}+s \cos \theta\right) \boldsymbol{i}+(s \sin \theta) \boldsymbol{j}
\end{gathered}
$$

The inertial velocity of the elevator in the rotating frame is given by

$$
\dot{\boldsymbol{r}}_{e}=[\dot{d} \cos \theta-d(\dot{\theta}+\omega) \sin \theta] \boldsymbol{i}+\left[\dot{d} \sin \theta+d \dot{\theta} \cos \theta+\omega\left(R_{E}+d \cos \theta\right)\right] \boldsymbol{j}
$$

with similar expressions for the counterweight and arbitrary point along the ribbon. Note, however, that the radial velocity terms $\dot{l}$ and $\dot{s}$ do not appear in these equations because the ribbon length is assumed to be constant. It may be possible to control the ribbon oscillations by reeling the main elevator ribbon, but this possibility is not considered here.

The total kinetic energy of the system is calculated by

$$
T=\frac{1}{2} m_{c} \dot{\boldsymbol{r}}_{c} \cdot \dot{\boldsymbol{r}}_{c}+\frac{1}{2} m_{e} \dot{\boldsymbol{r}}_{e} \cdot \dot{\boldsymbol{r}}_{e}+\frac{1}{2} \int_{0}^{l} \rho(s) \dot{\boldsymbol{r}}(s) \cdot \dot{\boldsymbol{r}}(s) \mathrm{d} s
$$

The potential energy due to the Newtonian gravitational field of the Earth is calculated as 


$$
\begin{aligned}
V & =-\frac{\mu m_{c}}{\sqrt{\left(R_{E}+l \cos \theta\right)^{2}+l^{2} \sin ^{2} \theta}}-\frac{\mu m_{e}}{\sqrt{\left(R_{E}+d \cos \theta\right)^{2}+d^{2} \sin ^{2} \theta}} \\
& -\int_{0}^{l} \frac{\mu \rho(s)}{\sqrt{\left(R_{E}+s \cos \theta\right)^{2}+s^{2} \sin ^{2} \theta}} \mathrm{d} s
\end{aligned}
$$

where $\mu$ is the Earth's gravitational parameter. After application of Lagrange's equations, the equation of motion for the libration of the ribbon is given by

$$
\begin{aligned}
\ddot{\theta} & =-2 \frac{m_{e} d \dot{d}(\dot{\theta}+\omega)}{\mathrm{M}_{1}}-\frac{\mathrm{M}_{2}}{\mathrm{M}_{1}} \omega^{2} R_{E} \sin \theta+\frac{1}{\mathrm{M}_{1}} \mu R_{E} \sin \theta\left[\frac{m_{e} d}{\left(R_{E}^{2}+2 R_{E} d \cos \theta+d^{2}\right)^{3 / 2}}\right. \\
& \left.+\frac{m_{c} l}{\left(R_{E}^{2}+2 R_{E} l \cos \theta+l^{2}\right)^{3 / 2}}+\int_{0}^{l} \frac{\rho(s) s}{\left(R_{E}^{2}+2 R_{E} s \cos \theta+s^{2}\right)^{3 / 2}} \mathrm{~d} s\right]
\end{aligned}
$$

where

$$
\begin{gathered}
\mathrm{M}_{1}=m_{e} d^{2}+m_{c} l^{2}+\int_{0}^{l} \rho(s) s^{2} \mathrm{~d} s \\
\mathrm{M}_{2}=m_{e} d+m_{c} l+\int_{0}^{l} \rho(s) s \mathrm{~d} s
\end{gathered}
$$

The equations of motion for the elevator represent a damped nonlinear oscillator. The damping (positive and negative) is provided by movement of the elevator along the ribbon, $\dot{d}$. For all practical purposes, the libration angle of the ribbon will be small. Equation (7) can be linearized around the vertical position to obtain the natural frequency of the libration mode. The equation shows that there is a non-zero forcing contribution of the elevator as it moves along the ribbon. Hence, any movement of the elevator along the ribbon initiates libration of the ribbon. Such libration is undesirable as it increases the tension in the ribbon and can ultimately affect the accuracy of payload insertion into orbit. The next section examines methods of eliminating the residual libration after climbing the ribbon.

\section{B. Multibody Elevator Model}

The use of a simple model for the elevator system neglects the lateral oscillations of the ribbon. In smaller scale tethered satellite systems, such oscillations can usually be safely neglected during preliminary control design since their effect in the real system is often hard to predict. Furthermore, the tensions involved are usually very small. However, because of the extreme scale of the tethered elevator system, lateral oscillations of the ribbon are strongly coupled to the motion of the counterweight. Hence, any lateral oscillations induce libration of the entire ribbon and counterweight. For this reason, it is important to assess the degree of coupling between the elevator motion and the ensuing system librations. This is important in assessing the applicability of simplified models for the design of ascent/descent trajectories for the elevator.

A lumped mass tether model is used in this paper. The model was originally presented in [6], where it was used to simulate prescribed acceleration-constant speed-deceleration motion of the of the elevator to geostationary altitude. In this work, the elevator motion is specified via the output of the trajectory optimizer.

The equations of motion reference to a rotating reference frame for $N_{\max }$ tether elements are given by 


$$
\begin{aligned}
\ddot{x}_{j}= & \frac{1}{m_{j}}\left(F_{x_{j}}^{s}+F_{x_{j}}^{d}+F_{x_{j}}^{g}\right)-\ddot{R}-\dot{\omega}_{y} z_{j}-2 \omega_{y} \dot{z}_{j}+\dot{\omega}_{z} y_{j}+2 \omega_{z} \dot{y}_{j}-\omega_{y} \omega_{x} y_{j}+\omega_{y}^{2} R \\
& +\omega_{y}^{2} x_{j}+\omega_{z}^{2} R+\omega_{z}^{2} x_{j}-\omega_{z} \omega_{x} z_{j} \\
\ddot{y}_{j}= & \frac{1}{m_{j}}\left(F_{y_{j}}^{s}+F_{y_{j}}^{d}+F_{y_{j}}^{g}\right)-\dot{\omega}_{z} R-\dot{\omega}_{z} x_{j}-2 \omega_{z} \dot{R}-2 \omega_{z} \dot{x}_{j}+\dot{\omega}_{x} z_{j}+2 \omega_{x} \dot{z}_{j}-\omega_{z} \omega_{y} z_{j} \quad j=1, \ldots, N_{\max } \\
& +\omega_{z}^{2} y_{j}+\omega_{x}^{2} y_{j}-\omega_{x} \omega_{y} R-\omega_{x} \omega_{y} x_{j} \\
\ddot{z}_{j} & =\frac{1}{m_{j}}\left(F_{z_{j}}^{s}+F_{z_{j}}^{d}+F_{z_{j}}^{g}\right)-\dot{\omega}_{x} y_{j}-2 \omega_{x} \dot{y}_{j}+\dot{\omega}_{y} R+\dot{\omega}_{y} x_{j}+2 \omega_{y} \dot{R}+2 \omega_{y} \dot{x}_{j}-\omega_{x} \omega_{z} R \\
& -\omega_{x} \omega_{z} x_{j}+\omega_{x}^{2} z_{j}+\omega_{y}^{2} z_{j}-\omega_{y} \omega_{z} y_{j}
\end{aligned}
$$

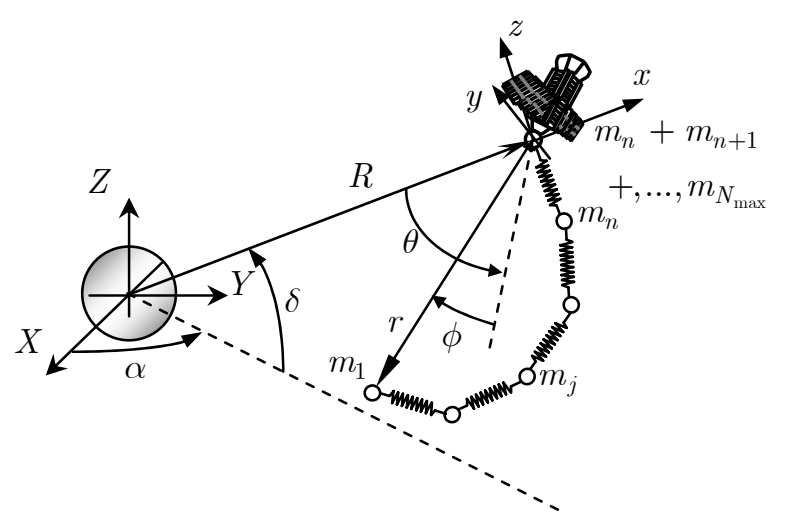

Fig. 2. Lumped mass tether model.

where the coordinates used are defined in Fig. 2 and

$$
\begin{aligned}
\boldsymbol{\omega} & =\left\{\begin{array}{lll}
\omega_{x}, & \omega_{y}, & \omega_{z}
\end{array}\right\}= \begin{cases}\dot{\alpha} \sin \delta, & -\dot{\delta}, \quad \dot{\alpha} \cos \delta\end{cases} \\
\dot{\boldsymbol{\omega}} & =\left\{\begin{array}{ll}
\dot{\omega}_{x}, & \dot{\omega}_{y}, \quad \dot{\omega}_{z}
\end{array}\right\} \\
& =\{\ddot{\alpha} \sin \delta+\dot{\alpha} \dot{\delta} \cos \delta, \quad-\ddot{\delta}, \quad \ddot{\alpha} \cos \delta-\dot{\alpha} \dot{\delta} \sin \delta\}
\end{aligned}
$$

Fig. 3 illustrates the discretization of the tethered elevator system to enable prescribed motion of the elevator. The complete system is split into two separate subsystems: an upper tether and a lower tether. For convenience, these have been labeled as tether [1] and [2], respectively. The motion of the elevator is achieved by changing the lengths of the tether segments closest to the elevator. The elevator mass is numbered as $m_{N_{\max }}$, which represents the maximum number of tether elements in either the upper or lower tether. The elements in the upper and lower tether subsystems are numbered in such a way that the extreme elements start at 1 and increase to $n_{1}$ and $n_{2}$, respectively. The key forces on the system are tension, damping, and gravity. These forces are derived explicitly in [6].

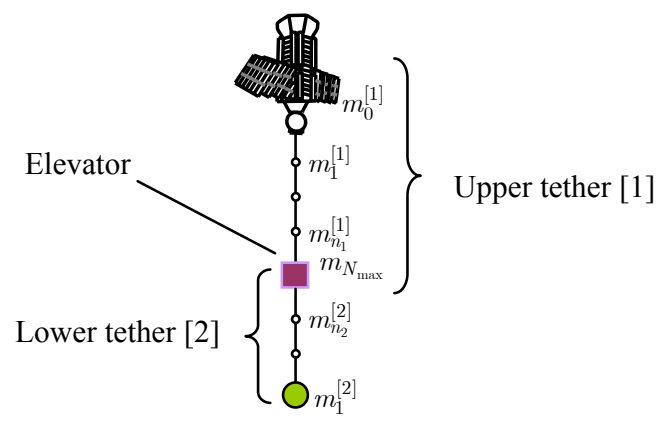

Fig. 3. Discretization of tethered elevator for prescribed elevator motion. 


\section{The Need for Elevator Speed Optimization}

Fig. 4 shows the results of a simulation of a $1000 \mathrm{~kg}$ elevator climbing a 70,000 km long ribbon to geostationary altitude. The elevator moves at a constant speed of $50 \mathrm{~m} / \mathrm{s}$, with acceleration and decelerations of $4 \mathrm{~m} / \mathrm{s}^{2}$ at the beginning and end of the journey. The major effect of the motion on the dynamics is the initialization of the first libration mode. Note that the first two modes of the system are the rigid body oscillations, about the anchored tip, shown in Fig. 5. The first mode is in the plane of the orbit, whereas the second mode is out of the orbital plane. There is almost no damping in the system and hence the libration continues well after the elevator ceases climbing. Rather than climbing at a constant velocity, a better strategy is to control the elevator's ascent in such a way that the librational oscillations are minimized. However, it is not an intuitive design task - we utilize optimal control to obtain a rapid solution to this problem.
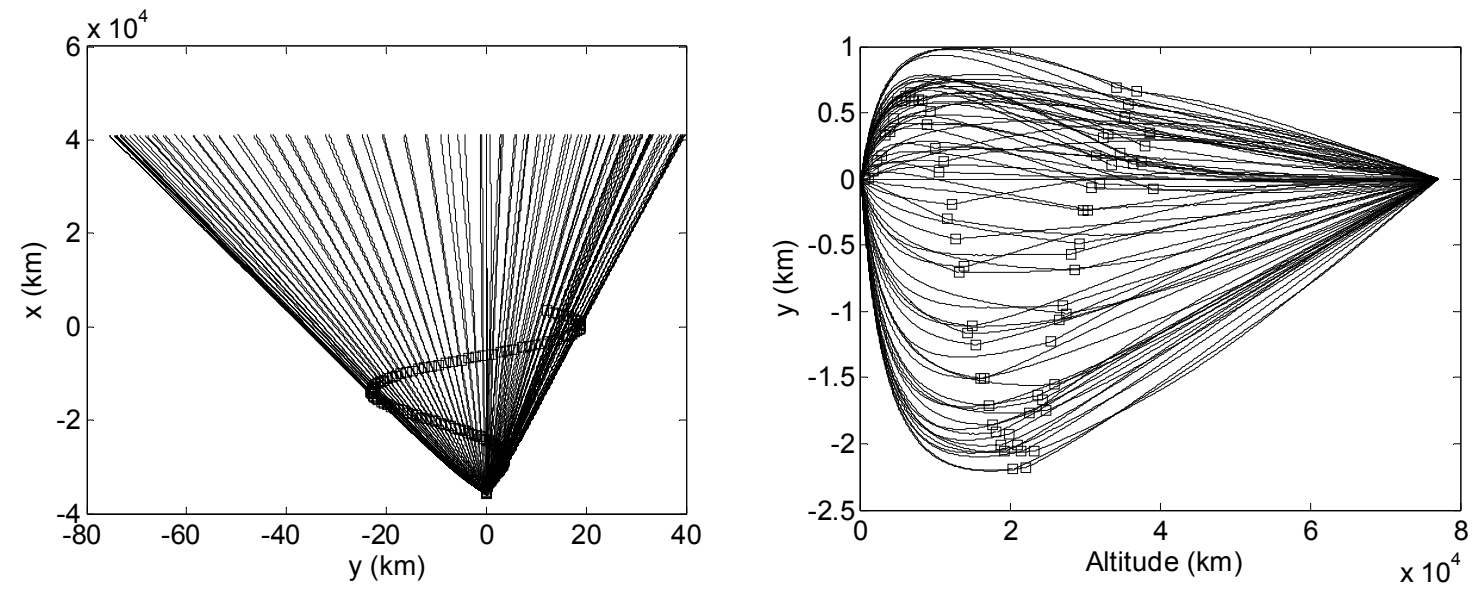

Fig. 4. Response of system to elevator climbing to geostationary altitude at $50 \mathrm{~m} / \mathrm{s}$.
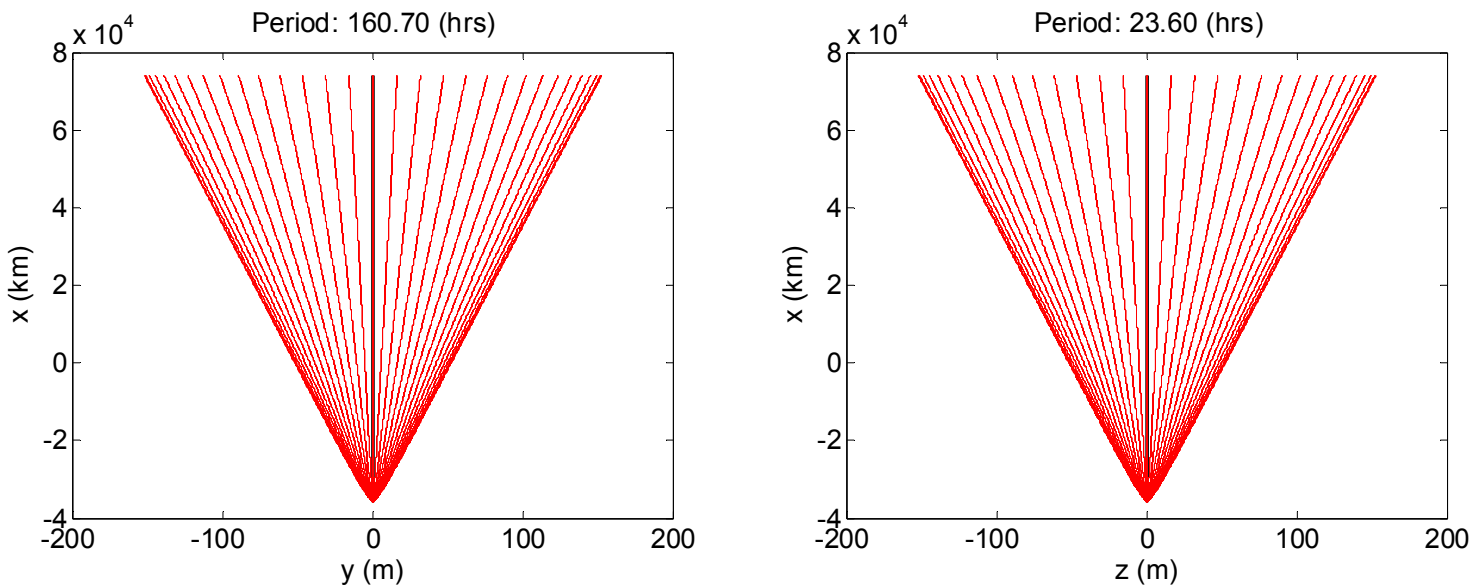

Fig. 5. Mode 1 and 2 for $100,000 \mathrm{~km}$ anchored tether.

\section{III.Optimization of Climber Motion}

In [7], Cohen and Misra analyzed the equations of motion for the ribbon libration under elevator forcing effects and proposed synchronizing the ascent of three climbers to null the effects of the residual ribbon libration at the end of the climb to geostationary altitude. This assumes that the climbers maintain constant speed during the climb. Any faults in the elevator's motors or disruption in power supply could result in failure of the method to eliminate the residual oscillations. It is of interest to be able to plan the ascent of a single climber to geostationary altitude such that the final ribbon libration is nullified. This is not possible by maintaining a single climber at constant speed. The advantage of optimizing the motion of a single climber is that it is possible to replan trajectories in case of motor failures or other unscheduled events. Furthermore, coordinating multiple climbers could be a logistical issue and would not be optimal from an operational perspective. 


\section{A. Numerical Optimization of Elevator Ascent}

\section{Basic Ascent Problem}

Consider the problem of finding the optimal climber acceleration $u(\cdot)=\ddot{d}(\cdot)$ to minimize the cost function

$$
J=\int_{t_{0}}^{t_{f}}\left(u^{2}+c_{1} x_{4}^{2}\right) \mathrm{d} t
$$

subject to the equations of motion

$$
\begin{aligned}
& \dot{x}_{1}=x_{2} \\
& \dot{x}_{2}=\ddot{\theta} \\
& \dot{x}_{3}=x_{4} \\
& \dot{x}_{4}=u
\end{aligned}
$$

the initial conditions

$$
\left\{x_{1}, x_{2}, x_{3}, x_{4}\right\}_{t_{0}}=\{0,0,0,0\}
$$

and the terminal constraints

$$
\left\{x_{1}, x_{2}, x_{3}, x_{4}\right\}_{t_{f}}=\left\{0,0, h_{G}, 0\right\}
$$

If a solution to the problem defined by Eqs. (13) through (16) exists, then it is possible to nullify the libration of the ribbon through proper scheduling of the elevator's speed on the ribbon. Note that the cost function minimizes a weighted functional of the climber acceleration and climber speed, where $c_{1}$ is a weighting parameter.

\section{Time Optimal Ascent}

An important class of maneuvers that can be used to establish lower bounds on the elevator's ascent is the minimum time maneuver. In such a maneuver, both the elevator acceleration and velocity are bounded to maximum permissible levels, and the cost function is selected as the final time,

$$
J=t_{f}
$$

The cost function is subject to the constraints

$$
\begin{aligned}
& \dot{d}_{\min } \leq \dot{d} \leq \dot{d}_{\max } \\
& \ddot{d}_{\min } \leq \ddot{d} \leq \ddot{d}_{\max }
\end{aligned}
$$

as well as the equations of motion defined by Eqs. (14). In addition, the maneuver is subject to the same initial and terminal constraints given in Eqs. (15) and (16).

\section{B. Numerical Solution Method}

An efficient solution to the nonlinear optimal control problem described in Section III.A can be obtained by application of direct transcription techniques combined with nonlinear programming. In this paper, the continuous 
optimal control problem is transcribed into a discrete parameter optimization problem by way of the Legendre pseudospectral method [12-13]. This process is automated in the software DIRECT [14].

\section{IV.Numerical Results}

\section{A. Time Optimal Ascent}

As noted in the previous section, it is important to establish a lower bound to the feasible maneuver time for realistic constraints on the elevator's maximum speed and acceleration. The time optimal ascent problem is solved for the case where the elevator is $1000 \mathrm{~kg}$, the ribbon is $100,000 \mathrm{~km}$ long with a maximum cross-sectional area of 10 $\mathrm{mm}^{2}$, and the material strength is $35 \mathrm{GPa}$ with density $1300 \mathrm{~kg} / \mathrm{m}^{3}$.

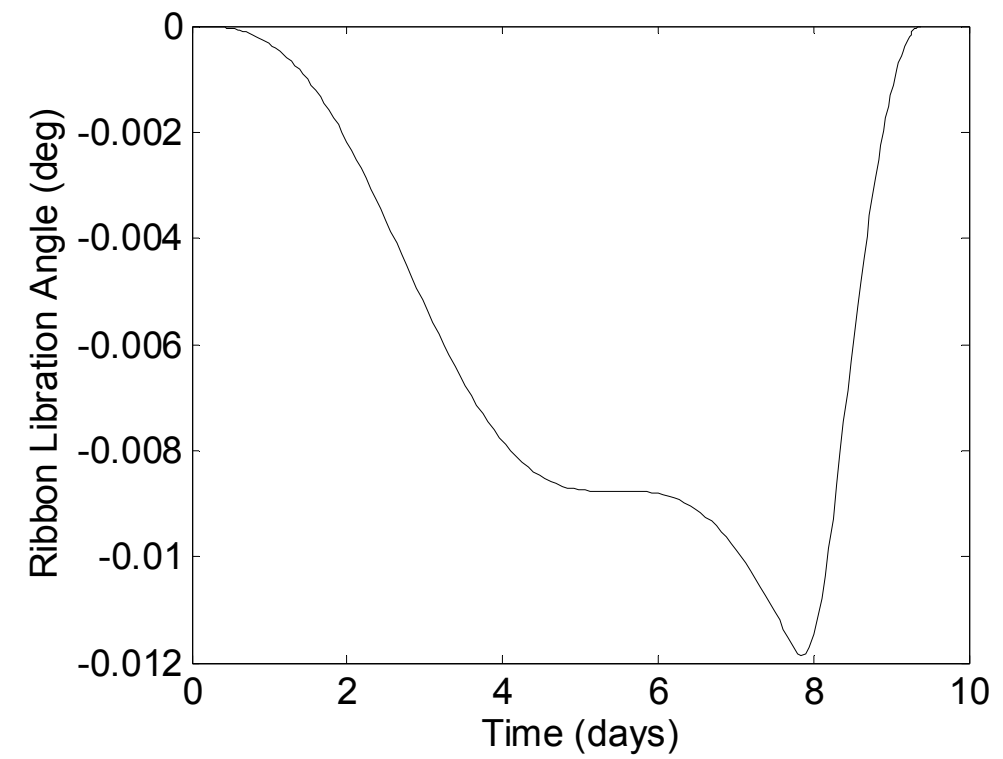

Fig. 6. Ribbon libration angle for time optimal elevator ascent to geostationary.

The optimal control problem is solved using the Legendre pseudospectral method with the time domain discretized into 200 nodes. The elevator's maximum speed is limited to $50 \mathrm{~m} / \mathrm{s}$, with a maximum acceleration of 4 $\mathrm{m} / \mathrm{s}^{2}$. The optimal solution is presented in Figs. 6 to 8. Fig. 6 shows that the libration angle of the ribbon peaks at approximately $0.012 \mathrm{deg}$ after approximately 8 days. The optimal time of ascent to nullify the ribbon libration is 9.383 days. Figs. 7 and 8 show that the elevator is able to maintain a constant speed throughout the vast majority of the ascent. Although hard to discern on the time scale, the initial acceleration and deceleration phases occur at the maximum limit. Fig. 8 shows that in order to bring the final libration angle and libration rate of the ribbon to zero at the final time, the elevator's speed must be reversed for just over half a day at about 8 days. At the higher altitude, the forcing effect is larger and is therefore able to force the ribbon swing back earlier than would have otherwise occurred. Once the swing back has commenced, the elevator is able to resume its course to geostationary, where it comes to a stop. Hence, the swing back is much faster than the initial swing away from the local vertical. 


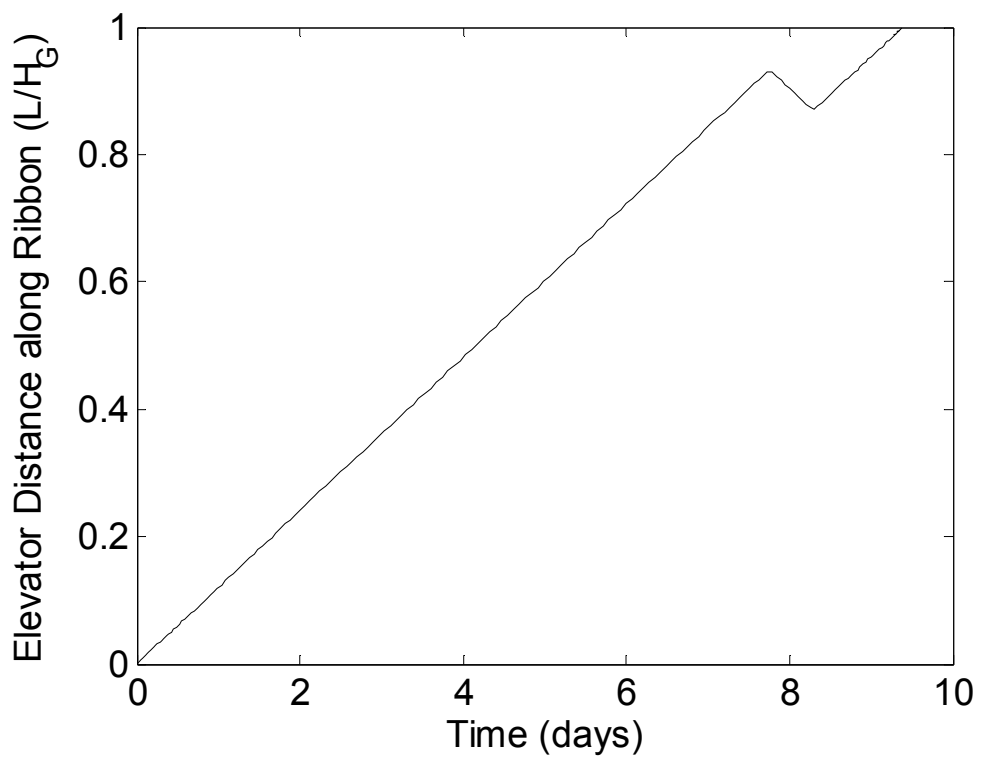

Fig. 7. Time optimal elevator ascent to geostationary.

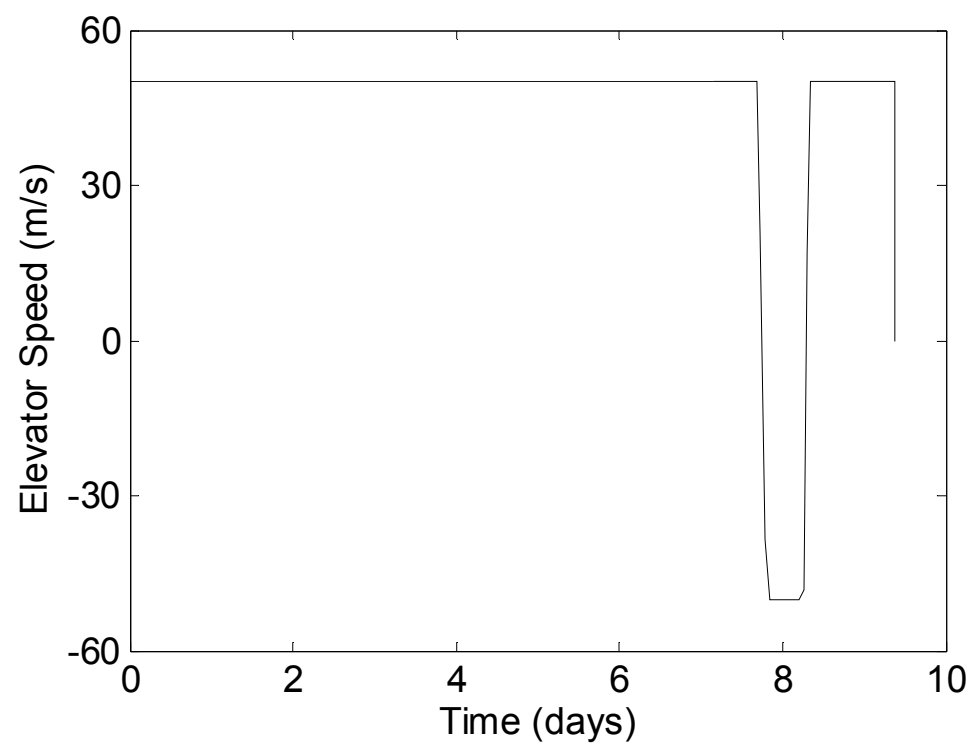

Fig. 8. Elevator speed schedule for time optimal ascent to geostationary altitude.

\section{B. Effect of Time of Ascent}

In this section, the acceleration and speed constraints on the elevator are lifted, and smooth unconstrained optimal solutions are sought as a function of different system parameters. In this particular subsection, the time of ascent is varied and the elevator's motion is optimized to eliminate the final ribbon libration. The time of ascent is varied from 4 days to 30 days in increments of 1 day. The elevator mass is $1000 \mathrm{~kg}$, the Young's modulus is 35 $\mathrm{GPa}$, the ribbon density is $1300 \mathrm{~kg} / \mathrm{m}^{3}$, the maximum cross-sectional area is $10 \mathrm{~mm}^{2}$, and the ribbon length is $100,000 \mathrm{~km}$.

The results of numerical optimizations are shown in Figs. 9 to 11. Fig. 9 shows the librational response of the ribbon to the elevator's motion for the optimized trajectories as a function of the time of travel. For clarity, the time scale is normalized by time of travel. For the fastest travel time, the libration angle of the ribbon reaches a peak just after the midway point. The optimal solution for eliminating the residual libration does not involve simply climbing at a constant speed. Instead, the speed peaks at approximately $15 \%$ of the trip time, then slowly decreases to zero. The elevator reverses direction after approximately $45 \%$ of the trip time, which causes the libration to reach its peak quickly. The elevator then accelerates back towards geostationary, reaching a second peak of $200 \mathrm{~m} / \mathrm{s}$, after which 
the elevator is slowed back to a stop. Increasing the transit time decreases the velocity peaks at the beginning and end of the transfer, and reduces the need for the elevator to travel back down the ribbon. Increasing the time of travel leads to a decrease in the peak libration amplitude. In addition, the libration peak shifts towards the end of the ascent. For very long travel times, the ribbon undergoes a series of low amplitude oscillations about a non-zero mean, culminating in a slightly larger peak just prior to returning to the local vertical.

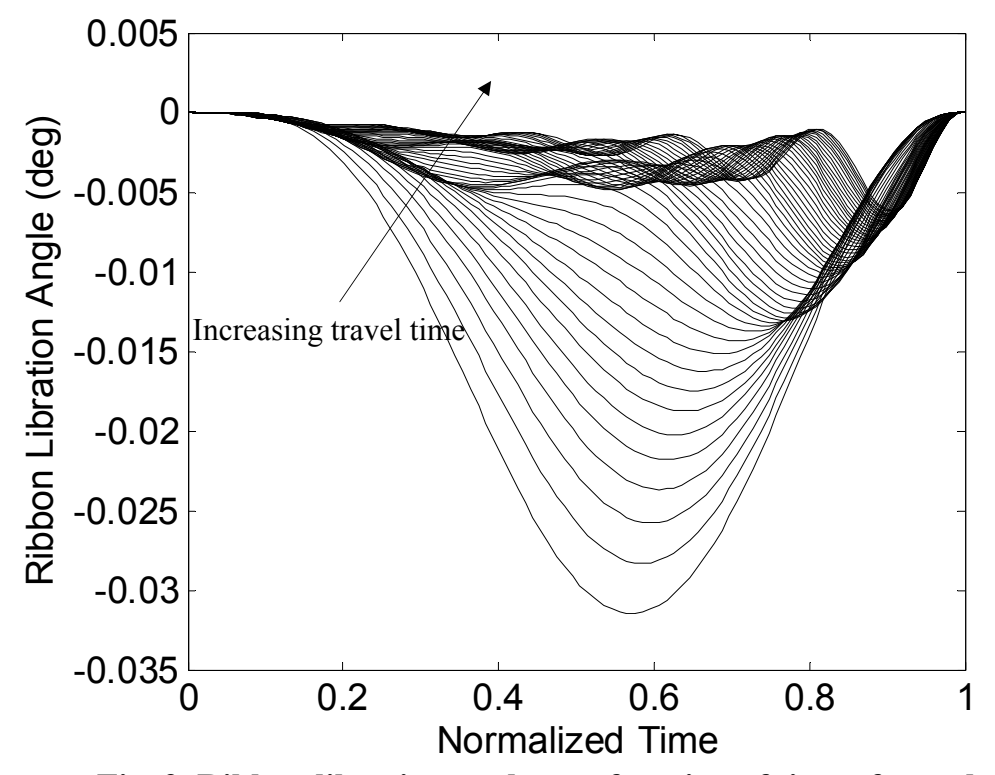

Fig. 9. Ribbon libration angle as a function of time of travel.

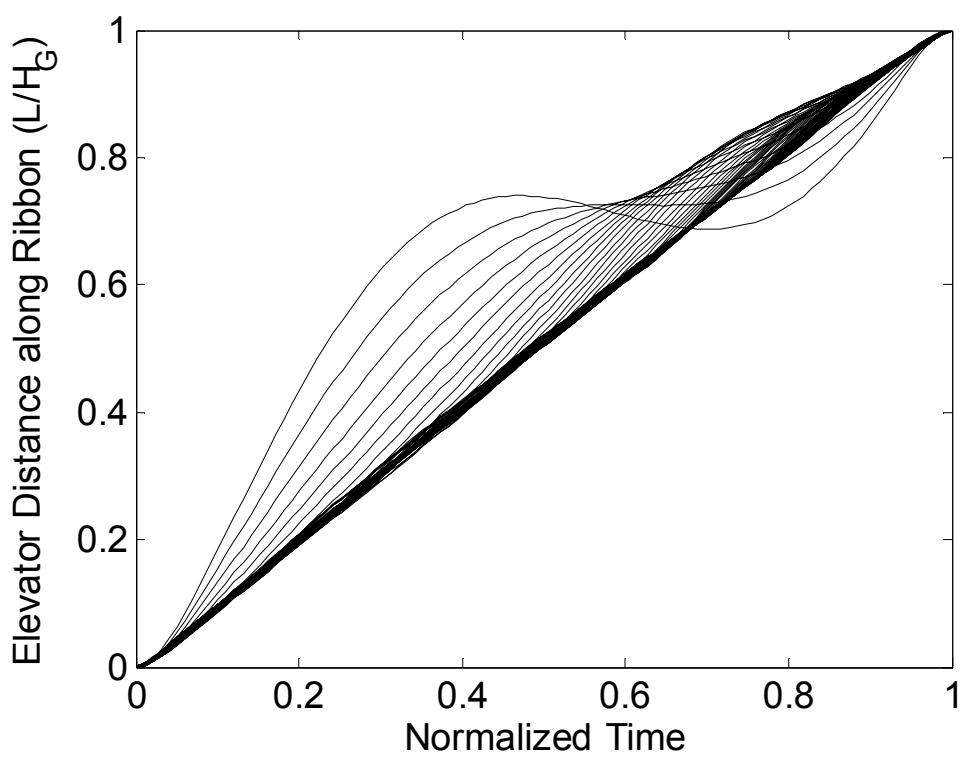

Fig. 10. Normalized distance of elevator along ribbon for optimal ascent. 


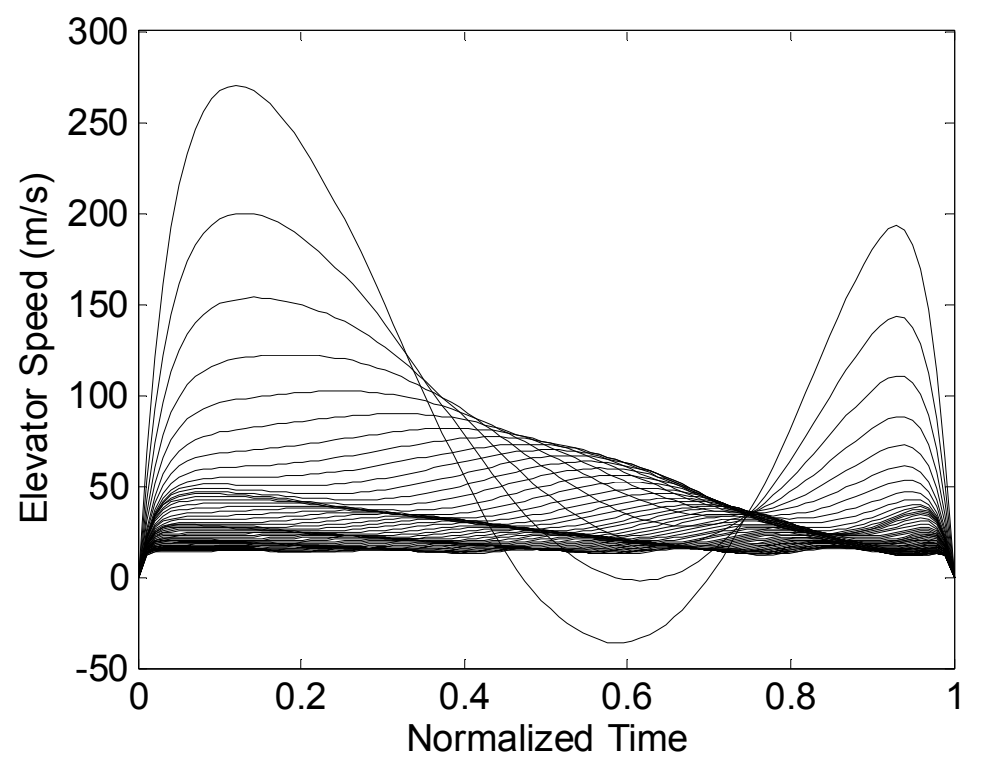

Fig. 11. Optimal elevator speed as a function of time of ascent.

\section{Effect of Elevator Mass}

The Coriolis forces exerted on the ribbon as a result of the elevator's motion along it are directly proportional to the elevator mass. Hence, the torque acting on the ribbon to disturb it away from the vertical position depends directly on the elevator mass. In this section, the same basic system is used to simulate the effects of elevator mass on the optimal climber trajectories. The ribbon is $100,000 \mathrm{~km}$ long with Young's modulus $35 \mathrm{GPa}$, the maximum cross-sectional area is $10 \mathrm{~mm}^{2}$, and mass density of $1300 \mathrm{~kg} / \mathrm{m}^{3}$. The travel time is fixed at 10 days, and the elevator mass is varied between 1000 and $20,000 \mathrm{~kg}$.

Optimal elevator trajectories are shown in Figs. 12 through 14. Fig. 12 shows that the peak ribbon angle increases in direct proportion to the elevator mass. This shape of the variation in ribbon angle is essentially the same, irrespective on the elevator mass. Initially the ribbon remains in vertical equilibrium and only starts to display noticeable deviations after approximately 1 day of travel. The libration peaks after approximately 7 days, after which the ribbon rapidly converges back to the stable equilibrium position. Figs. 13 and 14 show the variation of the elevator position along the ribbon and the elevator's speed, respectively. These figures indicate that the optimal control inputs are relatively insensitive to elevator mass. This is an important result because it means that the elevator load can be changed substantially without impacting the open-loop control. Note, however, that this conclusion is only valid within the assumption that the ribbon remains straight. Increasing the elevator mass will create larger Coriolis forces and hence induce larger lateral ribbon vibrations. This was established through simulations in [6] and the main result is summarized in Fig. 15, which shows that the maximum lateral deviation of the tether from the line connecting the anchor and the counterweight is linearly proportional to the elevator mass. 


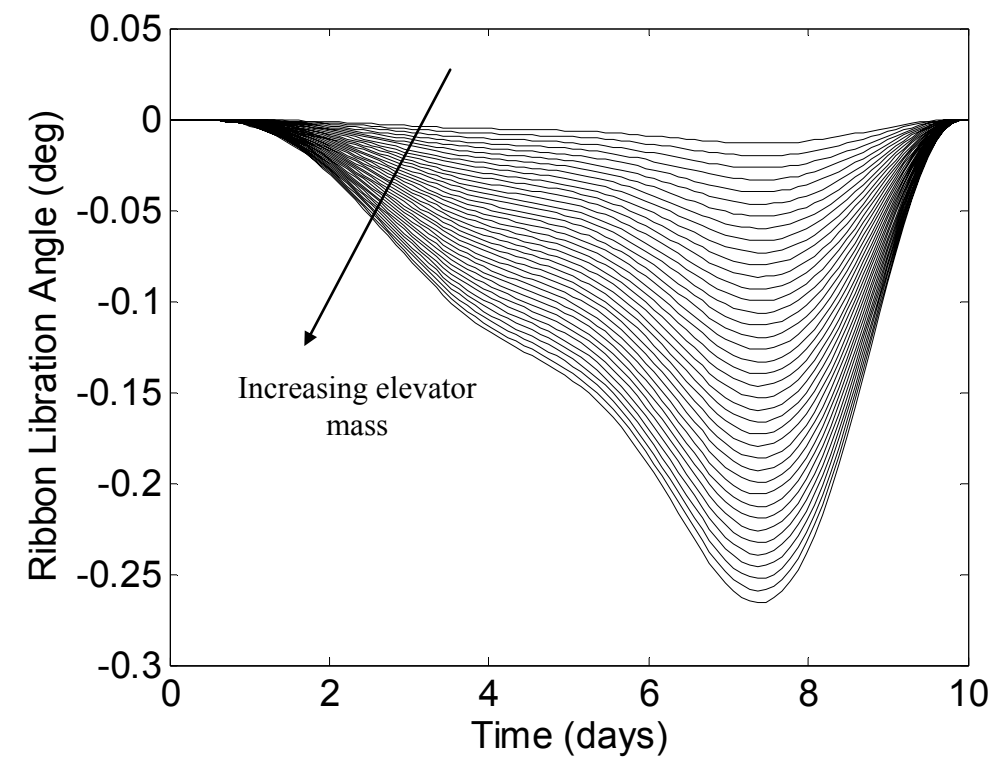

Fig. 12. Ribbon libration angle as a function of elevator mass.

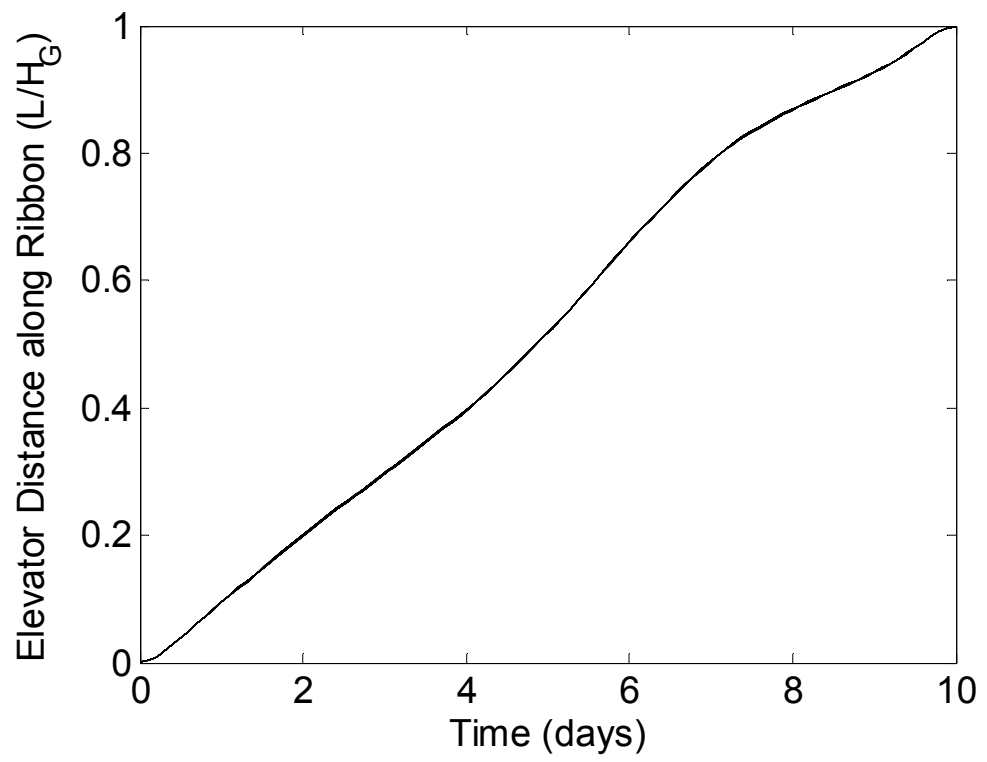

Fig. 13. Normalized distance of elevator along ribbon for optimal ascent as a function of elevator mass. 


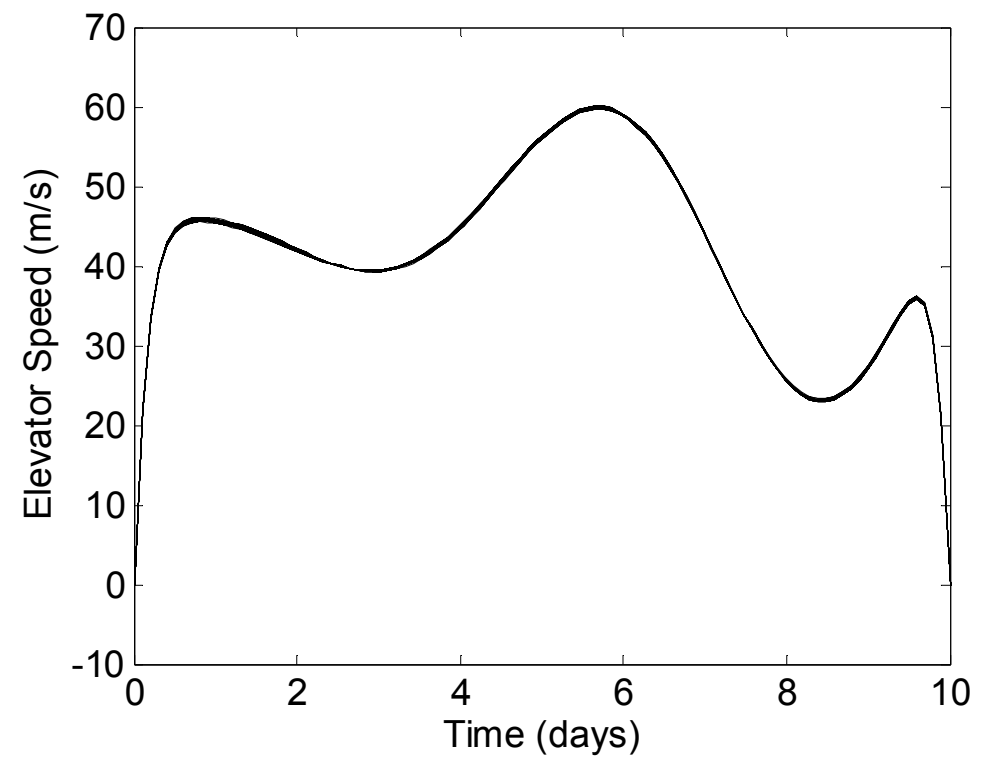

Fig. 14. Optimal elevator speed as a function of elevator mass.

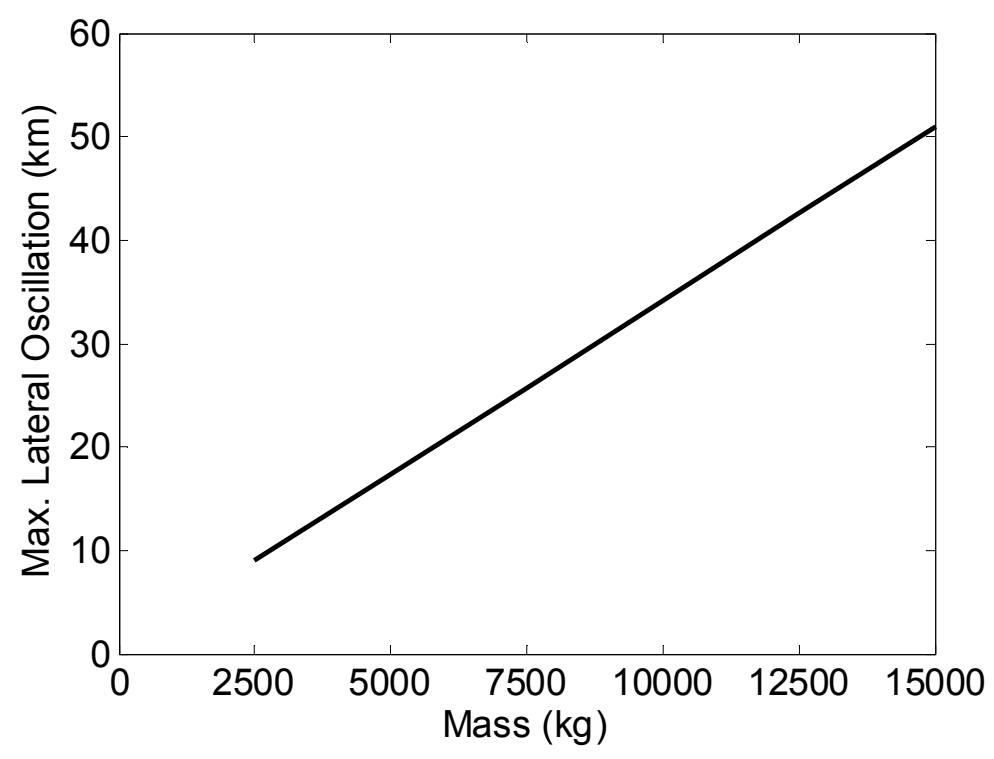

Fig. 15. Maximum lateral deflection of a flexible elevator ribbon during transit to geostationary altitude at a constant speed of $450 \mathrm{~m} / \mathrm{s}$.

\section{Effect of Tether Length}

The final parameter that is examined in this work is the ribbon length. Using the same parameters as in the previous example, but with an elevator mass of $1000 \mathrm{~kg}$, the ribbon length is varied between 70,000 and 160,000 $\mathrm{km}$. Results of the optimal trajectories are shown in Figs. 16 through 18. It should be noted that changing the ribbon length changes the total mass and mass distribution in the system. The results show, however, that changing the ribbon length has only a minor effect on the optimal trajectories. The effect is more substantial than changes in elevator mass. The trajectories are only affected because the system dynamics change due to the change in gravitational forces on the longer ribbon. In reality, the ribbon length will be virtually constant during operations. Hence, even changes on the order of a few hundred kilometers would have negligible impact on the control of the elevator. 


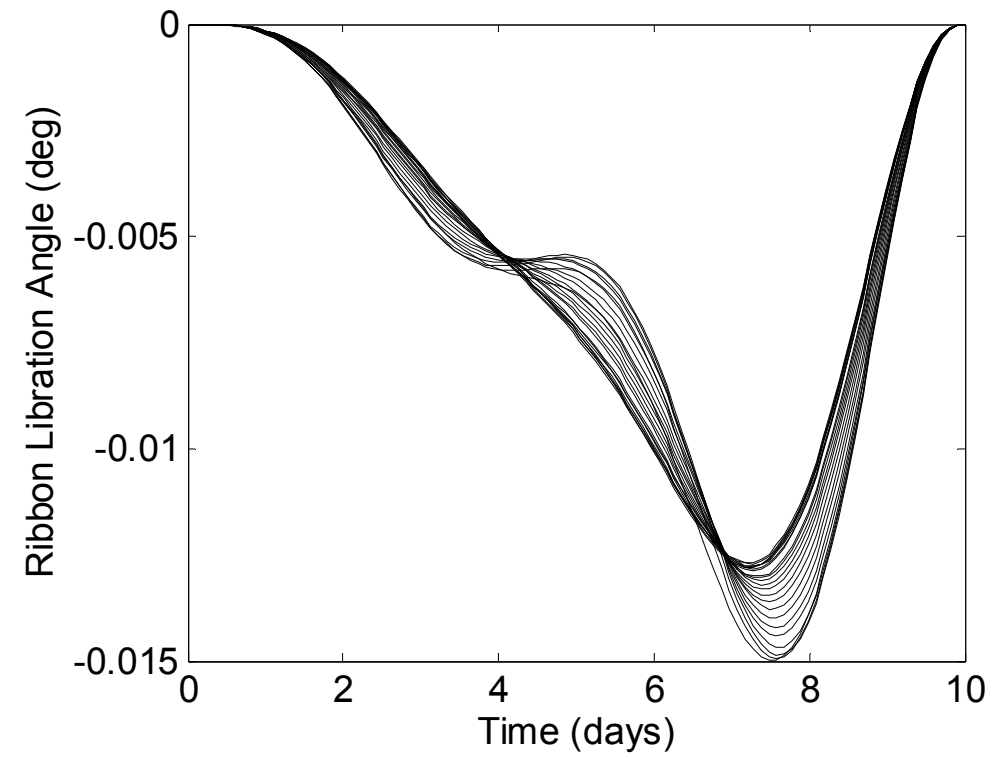

Fig. 16. Ribbon libration angle as a function of tether length.

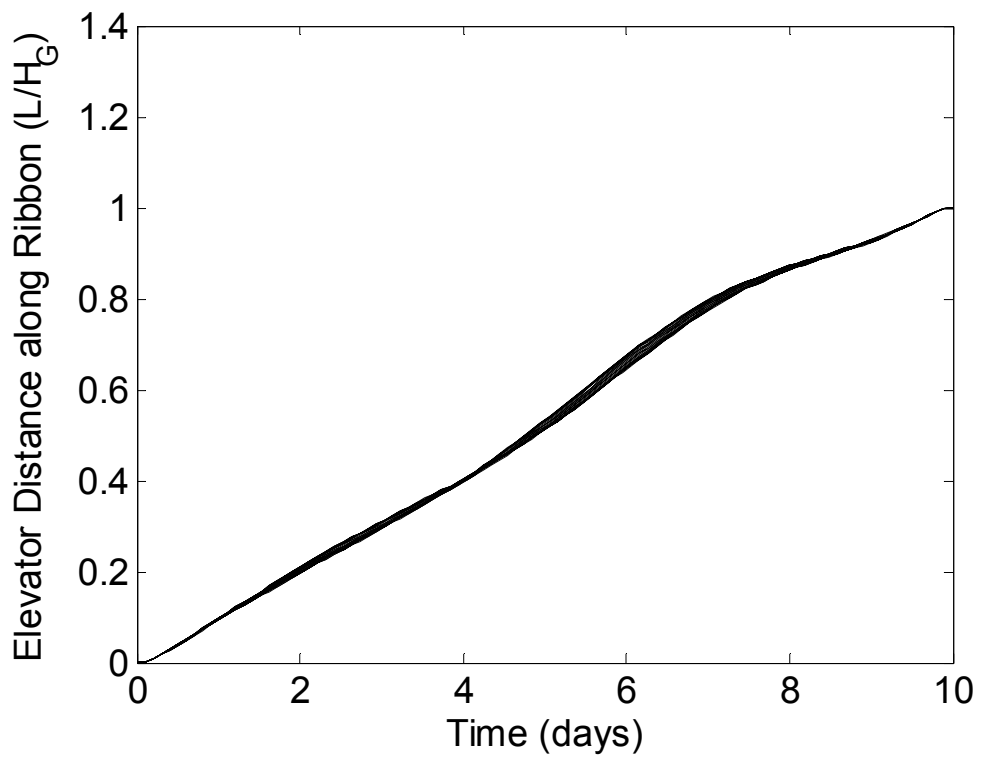

Fig. 17. Normalized distance of elevator along ribbon for optimal ascent as a function of tether length. 


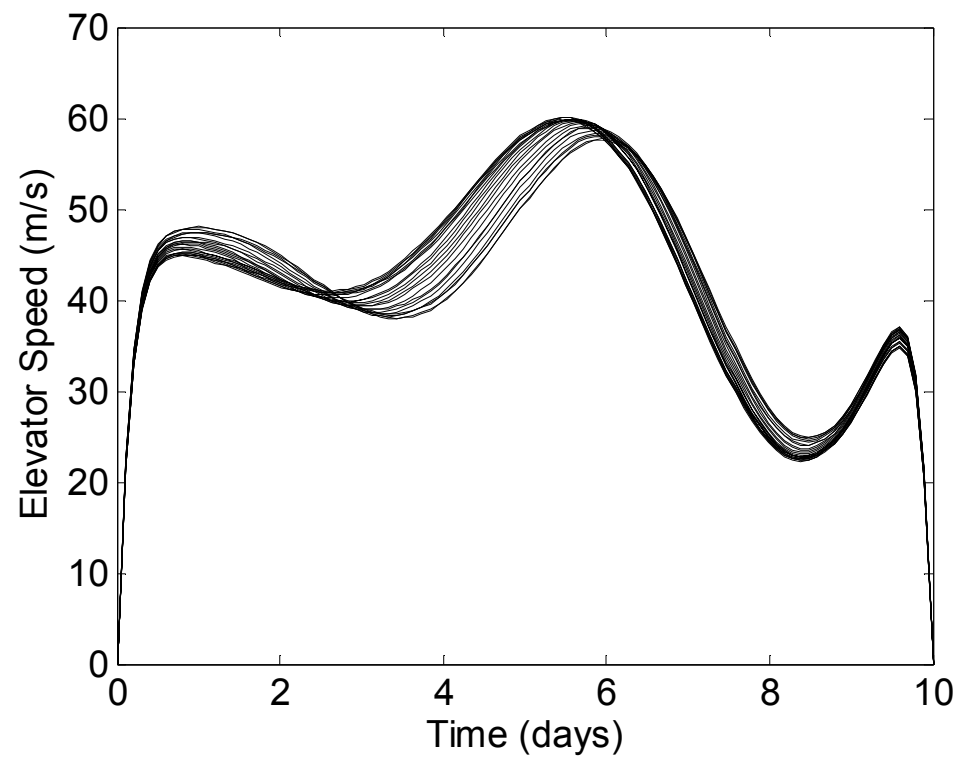

Fig. 18. Optimal elevator speed as a function of tether length.

\section{E. Numerical Simulation of an Optimal Trajectory in Flexible Ribbon Model}

The optimal solution was implemented in a flexible, elastic elevator model described in Section II. It has been found that the Young's modulus of the tether material plays a very important role in the dynamics. In particular, in order for the simple model to match the fundamental frequency of the multibody model, the Young's modulus must be very large (significantly larger than would be obtainable with Carbon nanotubes). An optimal solution was produced for a relatively short transit to Geostationary with a 100,000 km long ribbon. The maximum crosssectional area is $10 \mathrm{~mm}^{2}$ with a density of $1300 \mathrm{~kg} / \mathrm{m}^{3}$ and ultimate strength of $35 \mathrm{GPa}$. The reference optimal solution for transit to geostationary altitude for a $1000 \mathrm{~kg}$ climber is shown in Figs. 19 and 20. To implement the solution in the flexible model, the climber's velocity was interpolated. The libration angle of the flexible ribbon is shown in Fig. 21. This illustrates that the libration angle predicted using the simple model is quite different from that produced using the multibody model. The difference is almost two orders of magnitude, i.e., 100 times. This has a significant impact on the dynamics because the induced velocities are drastically different. However, the basic nature of the libration history is preserved, with the tether returning to an approximate vertical position. In addition, the deflection of the elevator from the ribbon centerline is approximately constant at $1 \mathrm{~km}$ throughout the climb to geostationary, as shown in Fig. 22. The lateral oscillations are also reduced by the optimal climber profile compared with a constant speed elevator ascent [6]. 


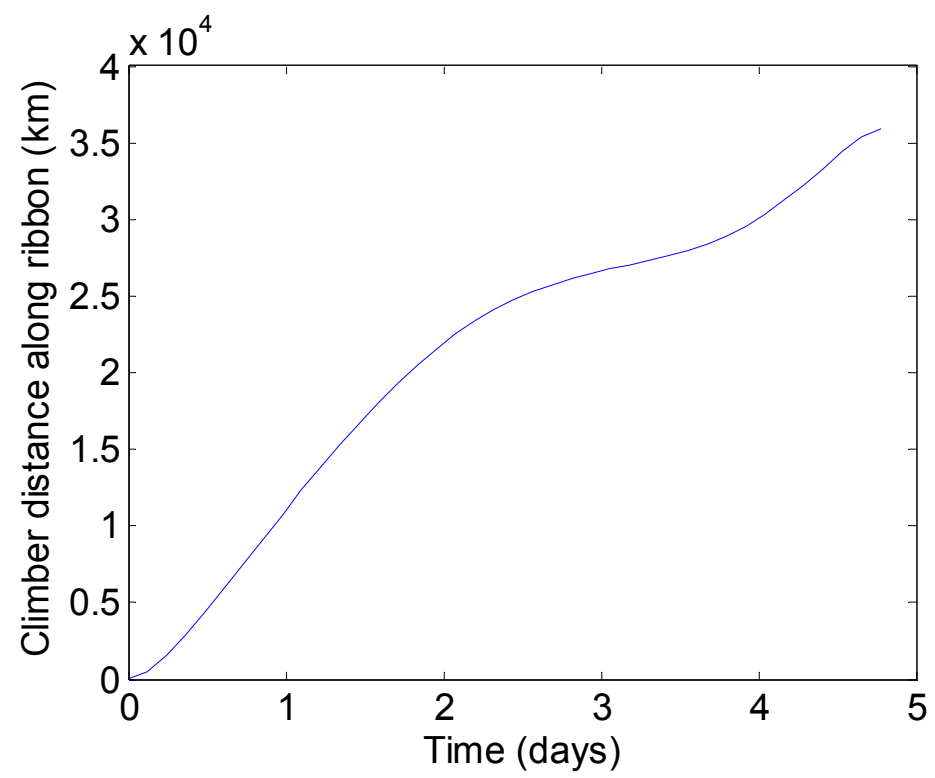

Fig. 19. Climber distance along ribbon for reference optimal solution.

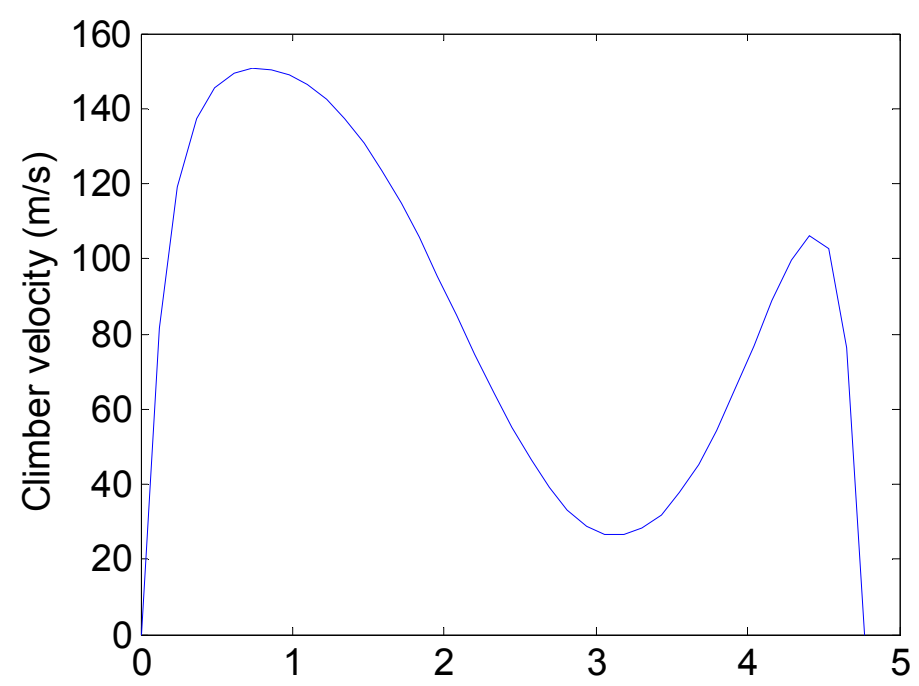

Fig. 20. Climber velocity for reference optimal solution. 


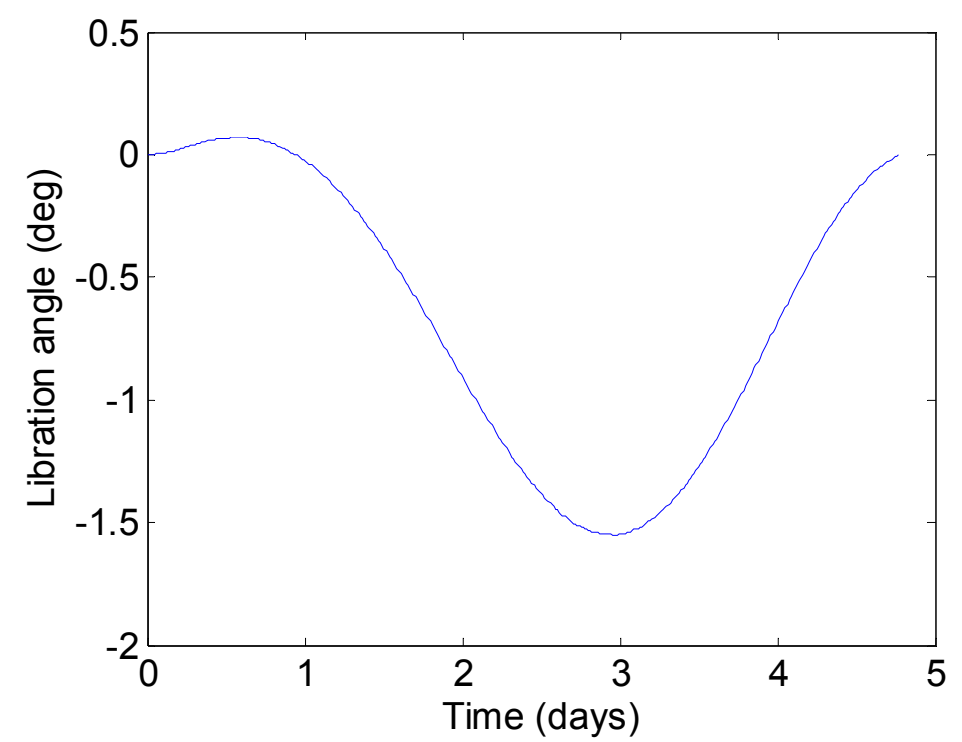

Fig. 21. Libration angle of ribbon using open-loop optimal solution implemented in flexible ribbon model.

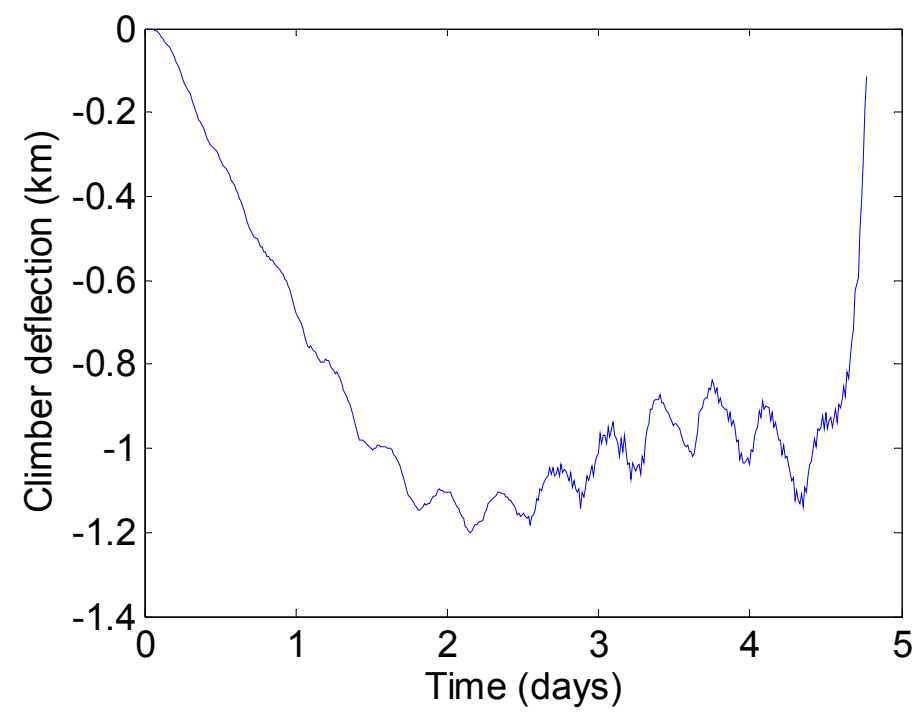

Fig. 22. Deflection of climber relative to line connecting anchor and counterweight for open-loop optimal solution.

\section{Conclusions}

The control of the ascent of a space elevator to geostationary altitude has been considered. The fundamental mode of vibration of an anchored ribbon is a rigid body oscillation about the anchor point on the Earth's surface. This mode leads to large oscillations of the counterweight, which can be located up to $100,000 \mathrm{~km}$ above the anchor point. As the elevator climbs the ribbon, Coriolis forces create a torque that initiates the librational mode. The lack of damping in space causes this mode to persist. Using a simplified model that only accounts for this mode, it is possible to solve the ascent problem using optimal control. The results show that it is possible to eliminate the librational oscillations by reversing the direction of the elevator for a short time. For a maximum speed of $50 \mathrm{~m} / \mathrm{s}$, with accelerations limited to $4 \mathrm{~m} / \mathrm{s}^{2}$, the optimal trip time is on the order of 9.38 days. 


\section{References}

1. Tsiolkovsky, K.E., Speculations Between Earth and Sky, Isd-vo AN-SSSR, Moscow, 1895, p. 35 (In Russian, reprinted in 1959).

2. Pearson, J., "The Orbital Tower: A Spacecraft Launcher using the Earth's Rotational Energy," Acta Astronautica, Vol. 2, 1975, pp.785-799.

3. Iijima, S., "Helical Microtubules of Graphitic Carbon," Nature, Vol. 354, 1991, pp. 56-58.

4. Cohen, S.S., and Misra, A.K., "Elastic Oscillations of the Space Elevator Ribbon," Journal of Guidance, Control, and Dynamics, Vol. 30, No. 6, 2007, pp.1711-1717.

5. Edwards, B.C., and Westling, E.A., The Space Elevator: A Revolutionary Earth-to-Space Transportation System, 2003.

6. Williams, P., "Dynamic Multibody Modeling for Tethered Space Elevators," 58th International Astronautical Congress, Hyderabad, 24-28 Sept. 2007, IAC-07-D4.1.03.

7. Cohen, S.S., and Misra, A.K., "The Effect of Climber Transit on the Space Elevator Dynamics," 58th International Astronautical Congress, Hyderabad, 24-28 Sept. 2007, IAC-07-D4.1.04.I

8. Williams, P., and Blanksby, C., "Study on Prolonged Rendezvous using Tethers," AAS/AIAA Astrodynamics Specialist Conference, Aug. 7-11 2005, Embassy Suites Hotel, Lake Tahoe Resort, Paper AAS 05-321.

9. Williams, P., and Blanksby, C., "Prolonged Payload Rendezvous using a Tether Actuator Mass", Journal of Spacecraft and Rockets, Vol. 41, No. 5, 2004, pp.889-892.

10. McInnes, C.R., "Dynamics of a Particle Moving Along an Orbital Tower," Journal of Guidance, Control and Dynamics, Vol. 28, No. 2, 2005, pp. 380-382.

11. Fujii, H.A., Watanabe, T., Kusagaya, T., Sato, D., and Ohta, M., "Dynamics of a Flexible Space Tether Equipped with a Crawler Mass," Journal of Guidance, Control, and Dynamics, Vol. 31, No. 2, 2008, pp.436440.

12. Elnagar, J., Kazemi, M.A., and Razzaghi, M., "The Pseudospectral Legendre Method for Discretizing Optimal Control Problems," IEEE Transactions on Automatic Control, Vol. 40, No. 10, 1995, pp.1793-1796.

13. Ross, I.M., and Fahroo, F., "Legendre Pseudospectral Approximations of Optimal Control Problems," Lecture Notes in Control and Information Sciences, Vol. 295, Springer-Verlag, New York, pp.327-342.

14. Williams, P., "User's Guide to DIRECT Version 1.17," Technical Report, Melbourne, Australia, March 2005. 